\title{
A New Model of Ultracapacitors Based on Fractal Fundamentals
}

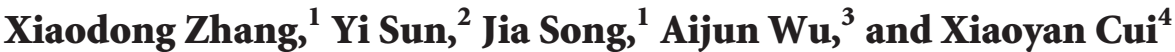 \\ ${ }^{1}$ School of Electrical Engineering, Beijing Jiaotong University, Beijing 100044, China \\ ${ }^{2}$ China Electrotechnical Society, Beijing 100823, China \\ ${ }^{3}$ Beijing Railway Bureau, Beijing 100860, China \\ ${ }^{4}$ Automation School, Beijing University of Posts and Telecommunications, Beijing 100876, China
}

Correspondence should be addressed to Xiaodong Zhang; xdzhang@bjtu.edu.cn

Received 24 January 2014; Accepted 25 June 2014; Published 23 July 2014

Academic Editor: Hongjie Jia

Copyright ( 2014 Xiaodong Zhang et al. This is an open access article distributed under the Creative Commons Attribution License, which permits unrestricted use, distribution, and reproduction in any medium, provided the original work is properly cited.

\begin{abstract}
An intuitive model is proposed in this paper to describe the electrical behavior of certain ultracapacitors. The model is based on a simple expression that can be fully characterized by five real numbers. In this paper, the measured impedances of three ultracapacitors as a function of frequency are compared to model results. There is good agreement between the model and measurements. Results presented in a previous study are also reviewed and the paper demonstrates that those results are also consistent with the newly described model.
\end{abstract}

\section{Introduction}

Smart grids and energy systems always need energy storage. Ultracapacitors represent an alternative to batteries for storing electrical energy and can help to compensate for the limited power density of batteries [1]. They resemble rechargeable batteries in terms of their ability to transport and store charge, but they employ a very different charge storage mechanism. Ultracapacitors store electric energy by accumulating and separating opposite charges physically, as opposed to batteries, which store energy chemically [2] Opposing charges are separated by an electrode/electrolyte interface, which is referred to as an electrochemical doublelayer. Compared to batteries, ultracapacitors have a much longer charge-discharge cycle life $[2,3]$. The power density of ultracapacitors is considerably higher than that of batteries, and the energy density is higher than that of electrolytic capacitors for power applications. Ultracapacitors can store a high level of energy in a small volume and release this energy in a powerful burst [4]; so they are useful in power electronic systems and applications (e.g., power systems, automotive, telecommunication, and military) that need to provide or absorb sudden current surges [5]. The power output of ultracapacitors is limited by their internal impedance.
The impedance needs to be identified and characterized in order to develop models for different applications. The development of these models requires measurements of their dynamic electrical behavior [2-4].

Often, ultracapacitors are modeled with simple $R C$ circuits as shown in Figure 1. Models like this are sufficient for well-defined and stable electrical signals; however, they do not accurately describe the electrical behavior of these devices in dynamic and high-power situations.

Practical ultracapacitor models are more complicated. Conway [6] described the charge storage mechanism as a Faradic pseudocapacitance involving a redox reaction of microporous transition metal hydrous oxides. Some $[3,7]$ have modeled the behavior of ultracapacitors using $R C$ transmission line equivalent circuits. The porous electrode is described by a line of $R$ and $C$ elements representing the elemental double layer capacitance and the respective electrolyte resistance at a particular pore depth. A more complete description of the porous electrode behavior was given by De Levie [8]. Gualous et al. [4] took into account the physics of the ultracapacitor and proposed an equivalent circuit that described the ultracapacitor electrical behavior with two $R C$ branches as shown in Figure 2. This model considers the nonlinear relationship between the capacitance 


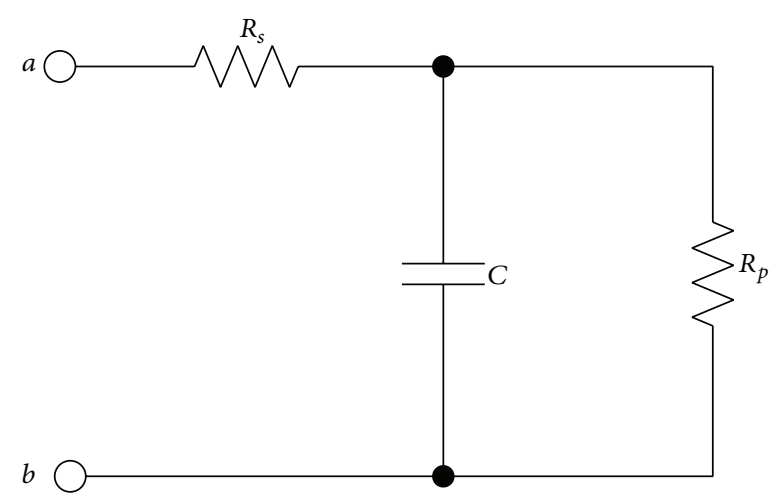

FiguRE 1: $R C$ circuit model for ultracapacitors.

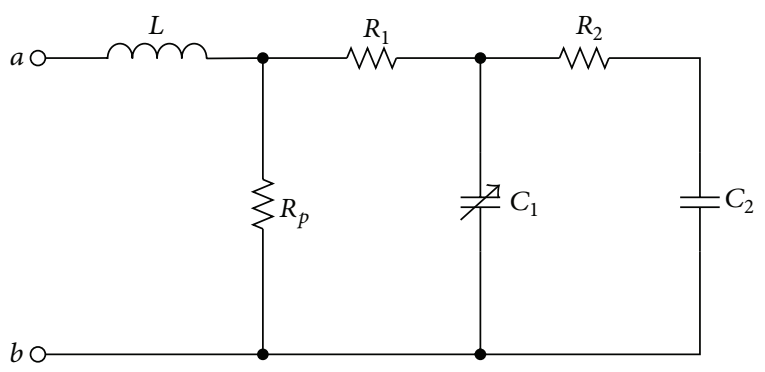

FIGURE 2: Equivalent circuit developed by Gualous et al.

of activated carbon particles and their surface area that varies with the type of activated carbon used and the way it is treated. But the model has six parameters to be identified and does not accurately describe ultracapacitor behavior at low frequencies.

Zubieta and Bonert [9] provided a model for the terminal behavior of an ultracapacitor based on physical reasoning. This model (see Figure 3) has three distinct $R C$ time constants. $R_{f}, C_{f}$ with the voltage-dependent capacitor $C_{f 2}$ $(F / V)$ which reflects the voltage dependence of the capacitance dominates the behavior of the ultracapacitor in the time over a period of seconds in response to a charge action. The other branches, $R_{l}$ with $C_{l}$ and $R_{m}$ with $C_{m}$, separately determine the terminal behavior in the range of minutes and the behavior for times longer than 10 mins. The resistor $R_{p}$ is a leakage resistor representing the self discharge property of the ultracapacitor. Others (e.g., [10]) have also developed equivalent circuit models based on variable time constants to fit the measured AC impedance data of ultracapacitors. In [10], the dependence of the resistance on frequency was divided into four distinct frequency zones and this model included a voltage-dependent capacitor as well.

However, the pores of activated carbon in an ultracapacitor have a complex branch pore structure for which any impedance analysis model should account. Furthermore, the parameters in the above models, especially the voltage dependent capacitance, can be difficult to quantify.

Buller et al. [11, 12] presented a model shown in Figure 4, which can be used to describe the behavior of ultracapacitors over a wide range of frequencies. $\underline{Z}_{p}$ is the complex pore

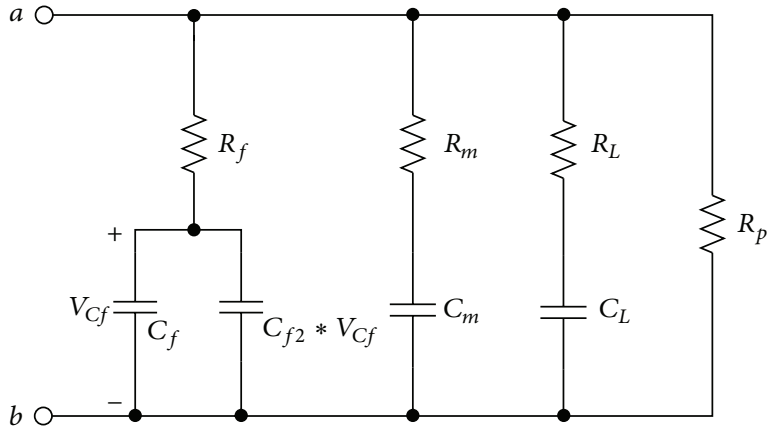

FIgURE 3: Equivalent circuit developed by Zubieta and Bonert.
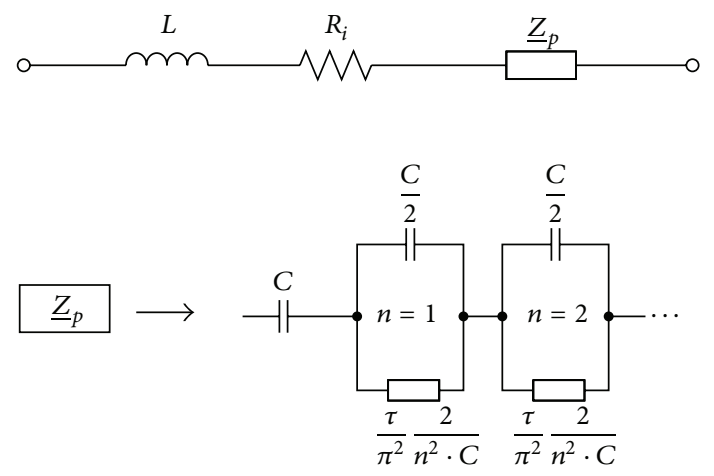

FIgURE 4: Equivalent circuit developed by Buller et al.

impedance related to the porosity of the ultracapacitor. The mathematical expression for $\underline{Z}_{p}$ is

$$
\underline{Z}_{p}(j \omega)=\frac{\tau \cdot \operatorname{coth}(\sqrt{j \omega \tau})}{C \cdot \sqrt{j \omega \tau}} .
$$

Unfortunately, this function is not well suited for circuit simulation software because of the coth term [13]. Moreover, when the number $N$ is high, the series denominators of the formula in Figure 4

$$
\frac{\tau}{\pi^{2}} \frac{2}{n^{2} \cdot C}
$$

become very large and may not be accurately determined by measurements. Buller et al. [12] used 10 cells and 20 parameters to model a $1400 \mathrm{~F}$ ultracapacitor.

$\mathrm{Qu}$ and Shi [7] proposed an $R C$-ladder network model for ultracapacitors based on the pore structure of activated carbon which is shown in Figure 5. This model is particularly intuitive, because it illustrates how more capacitance becomes available as the time constant of the charging cycle is increased. $R_{i}$ and $C_{i}(i=1, \ldots, n)$ can be treated as the resistance and capacitance of the pores with certain pore size. $R_{i} C_{i}$ gives the unit of time and indicates how fast the pore of certain size is.

Itagaki et al. [14] proposed a model for ultracapacitors based on a fractal pore structure with three sizes of cylindrical pores. This model consisted of resistors and complex impedances connected in a tree-like structure. 


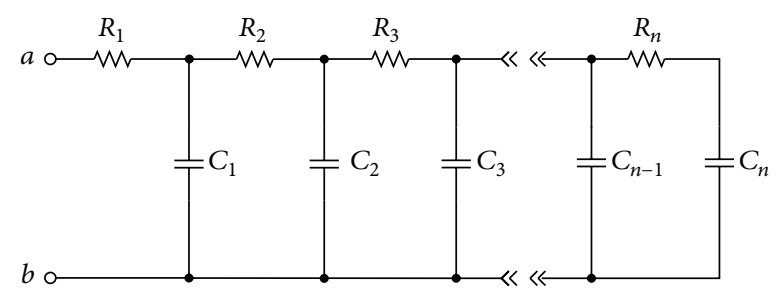

Figure 5: Equivalent circuit developed by Qu and Shi.

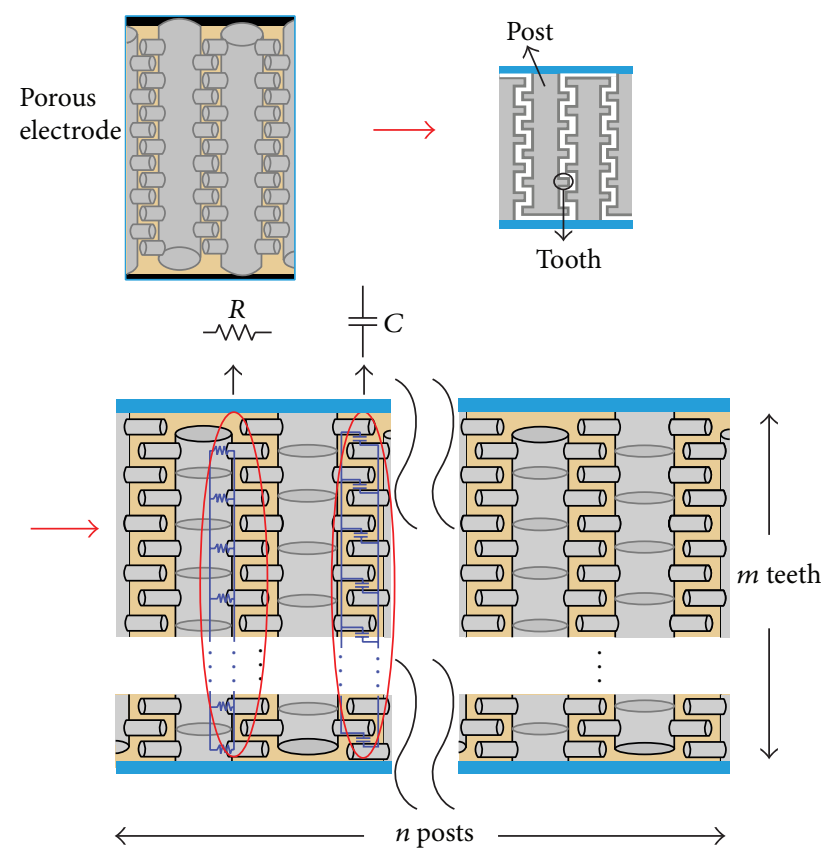

Figure 6: Pore model of the ultracapacitor electrodes.

This paper proposes a new electrical model for ultracapacitors that is also based on a fractal interpretation of their structure, but does not make any assumption about the size or shape of the pores. The new model is relatively simple and can be fully described by five parameters.

\section{Model Description}

Ultracapacitors are comprised of two highly porous activated carbon electrodes, which take surface area and charge separation distance to an extreme. The surface areas can be greater than 21,500 square feet per gram and the separation between the charged surfaces is reduced to distances on the order of nanometers [15]. The electrodes are immersed in a suitable electrolyte to facilitate the charge transfer and storage mechanism. Charges accumulate in the pores resulting in capacitance.

Consider the sample pore structure illustrated in Figure 6, a central conducting structure called a "post" is lined with many smaller structures referred to as "teeth." Half of the posts in this structure are connected to one electrode of an ultracapacitor and the other half are connected to the other electrode. The ultracapacitor has $2 n$ posts, and every post has

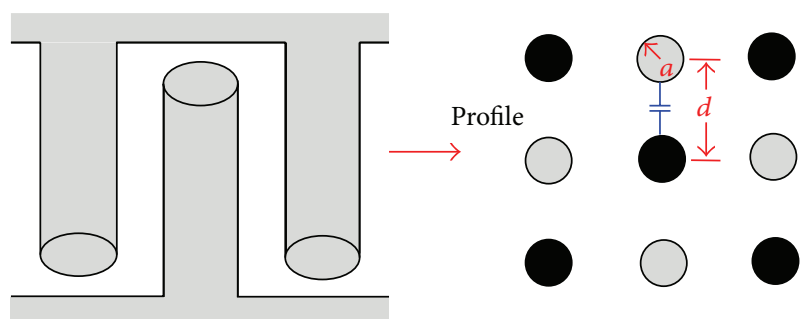

FIGURE 7: Capacitance scheme of porosity.

$m$ teeth. In this model, each tooth is a smaller version of the post that it is attached to.

If we start by analyzing the capacitance of the structure without any teeth, the total capacitance is $n$ times to the postto-post capacitance, because all of these capacitances are in parallel. The equivalent series resistance of the ultracapacitor is the resistance of one pair of posts divided by $n$, since the post resistances are also in parallel, therefore, an equivalent circuit for an ultracapacitor consisting of interleaved posts of uniform size would be a simple $R C$ circuit, where the value of $R$ is $R_{\text {post }} / n$ and the value of $C$ is $C_{\text {post }} \times n$. In this case, $R_{\text {post }}$ is the resistance associated with charge moving from one electrode into a single post and then to the second electrode. $C_{\text {post }}$ is the contribution to the capacitance of a single pair of posts. The time constant associated with charge populating the posts (i.e., the $R C$ time constant) is

$$
\tau=R C=\frac{R_{\text {post }}}{n} \times C_{\text {post }} \times n=R_{\text {post }} C_{\text {post }} .
$$

$R_{\text {post }}$ is proportional to the length of the post and inversely proportional to the cross-sectional area:

$$
R_{\text {post }}=\sigma \frac{l}{\pi \cdot A},
$$

where $\sigma$ is the electrical conductivity of the post, $l$ is the length of the post, and $A$ is the cross-sectional area of the post.

The capacitance, $C_{\text {post }}$, can be expressed as the capacitance between any two posts times a constant that is determined by the number of posts in proximity to a given post. A uniform post distribution is illustrated in Figure 7. $C_{\text {post }}$ is proportional to the length $l$ and inversely proportional to the natural $\log$ of the ratio of the post separation $d$, to the post radius $a$, as indicated below:

$$
C_{\text {post }}=k \frac{2 \pi \varepsilon l}{\ln (d / a)},
$$

where $\varepsilon$ is the permittivity of the dielectric.

A circuit model for the post-only ultracapacitor is provided in Figure 8.

Now, consider the structure of the intermeshed teeth between posts. In fractal geometry, each tooth would be a scaled down version of the post to which it was attached. If we assume that each post has $m$ teeth and the size of each tooth is $1 / m$ times the size of the post, then the resistance of a single tooth is

$$
R_{\text {tooth }}=\sigma \frac{l / m}{\pi A / m^{2}}=m \times R_{\text {post }} .
$$




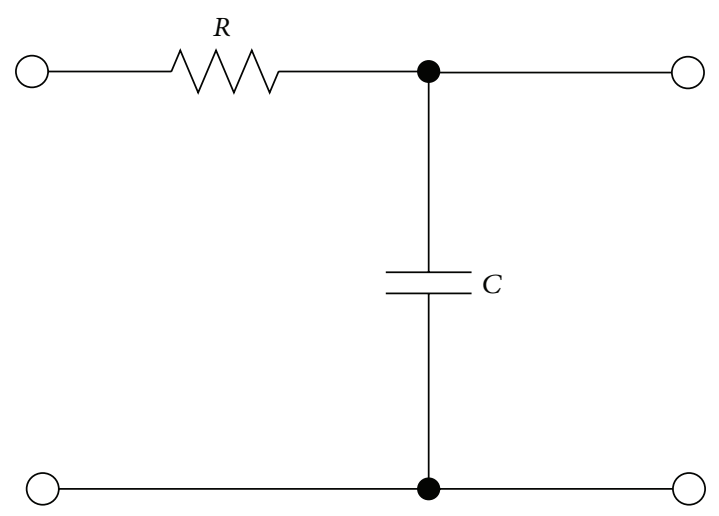

FIgURE 8: The post-only model.

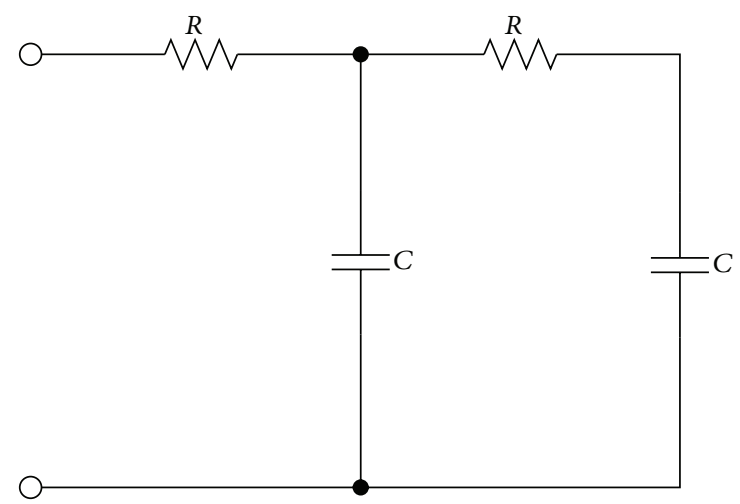

FIGURE 9: Equivalent circuit for a capacitor with one set of posts and teeth.

The capacitance, $C_{\text {tooth }}$, can be expressed as

$$
C_{\text {tooth }}=k \frac{2 \pi \varepsilon l / m}{\ln ((d / m) /(a / m))}=\frac{C_{\text {post }}}{m} .
$$

For $m$ teeth per post in parallel, the overall capacitance is multiplied by $m$ and the resistance is divided by $m$, yielding

$$
\begin{aligned}
R_{\text {teeth_per_post }} & =\frac{\left(m \times R_{\text {post }}\right)}{m}=R_{\text {post }}, \\
C_{\text {teeth_per_post }} & =\left(\frac{C_{\text {post }}}{m}\right) \times m=C_{\text {post }} .
\end{aligned}
$$

From (8) and (9), it is clear that the time constant associated with charge moving from the posts out onto the teeth is the same as the time constant associated with charge moving out onto the posts and can be calculated with (3). The equivalent circuit for the capacitor with both posts and teeth is shown in Figure 9. Both resistors in this model have the same value. Both capacitors also have the same value.

Employing (6) through (9), it is relatively straightforward to show that adding $m$ smaller teeth to each tooth in the structure shown in Figure 6 would result in the same amount of additional capacitance provided through the same amount of additional resistance. The fractal geometry with many layers of repeating structures would yield the equivalent

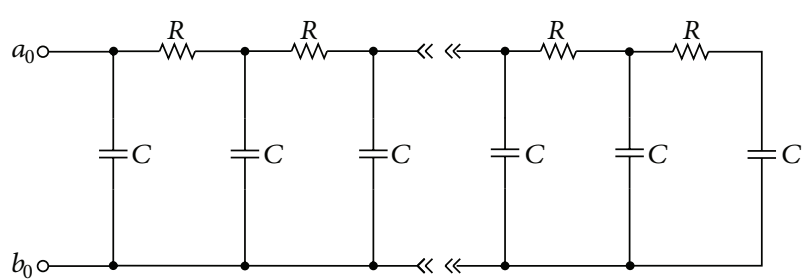

Figure 10: Electrical model of a fractal electrode structure.

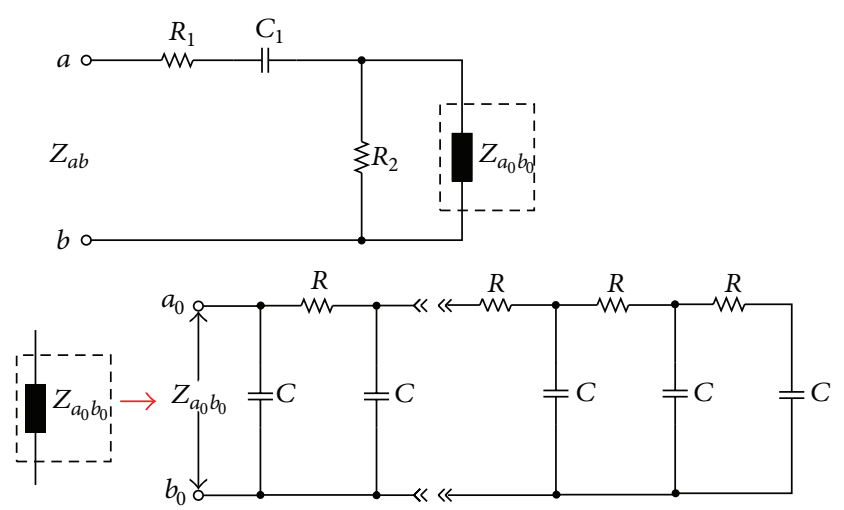

FIGURE 11: Ultracapacitor model described by only 5 real numbers.

circuit in Figure 10, where all of the resistors would have the same value and so do all of the capacitors.

Although the example in Figure 6 employs cylindrical pore structures, the resistance and capacitance will scale similarly with nearly any branch-like pore structure. Generally, the resistance of a branch will be proportional to its length and inversely proportional to its cross-sectional area. The capacitance between closely spaced branches will be proportional to its length and independent of cross-sectional area if the spacing between branches is also scaled. Therefore, the model in Figure 10 does not assume a particular pore structure as long as the successively smaller branches of the pores form fractal geometry.

As the different number of time between charging and discharging, the deterioration of ultracapacitors is also not the same. Few electrolytes will be decomposed to form the free insoluble product particles, which increase the resistance. Therefore, a resistor is paralleled in the model in Figure 10. The greater the loss is, the higher the resistor value will be.

Considering its ohmic resistance characteristics and ohmic capacitive characteristics, a resistor and a capacitor are in series; thus, the model in Figure 11 is proposed, which has 5 independent component values.

Although the model in Figure 11 has only 5 independent component values, it has an infinite number of elements. In order to develop a closed-form expression for the input impedance, it is convenient to use a Fourier transform technique. For the impedance ladder network in Figure 12, $Z_{n}$ is the equivalent input complex impedance and $Z_{n-1}$ is 


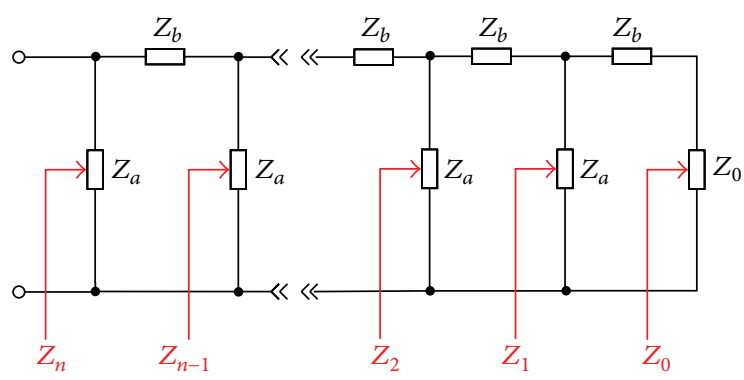

FIGURE 12: Complex impedance ladder network.

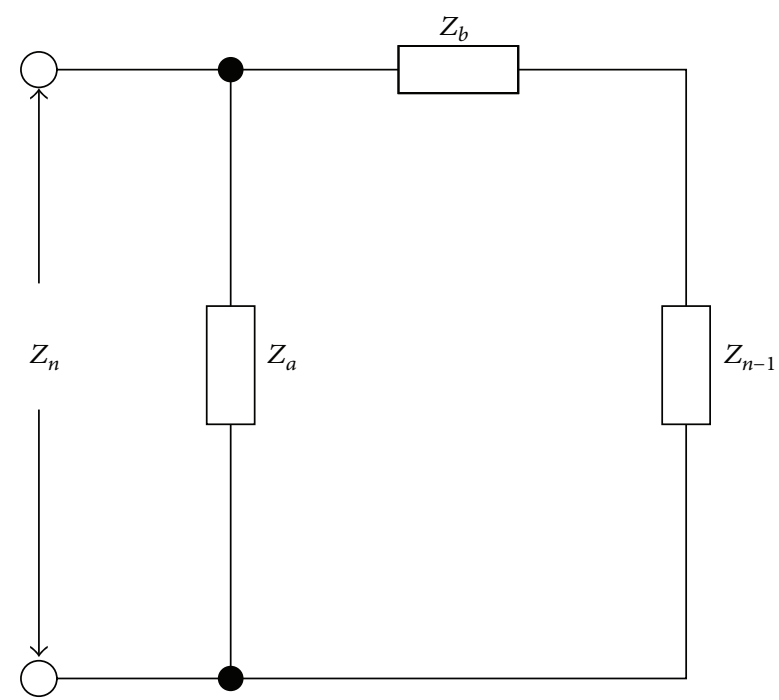

FIGURE 13: The equivalent circuit of ladder network in Figure 12.

the equivalent input complex impedance of the $(n-1)$-order ladder network (see Figure 13). So

$$
Z_{n}=\left(Z_{b}+Z_{n-1}\right) / / Z_{a} .
$$

The recurrence formula for $Z_{n}$ is

$$
Z_{n}=\frac{\left(Z_{a}\right) \cdot Z_{n-1}+\left(Z_{a} \cdot Z_{b}\right)}{Z_{n-1}+\left(Z_{a}+Z_{b}\right)}
$$

The solution of (11) can be obtained using a method presented in $[16,17]$. In this case, the coefficient matrix of $Z_{n}$ which is expressed by $Z_{n-1}$ is shown as follows:

$$
\begin{gathered}
A_{1}=\left[\begin{array}{cc}
Z_{a} & Z_{a} \cdot Z_{b} \\
1 & Z_{a}+Z_{b}
\end{array}\right], \\
A_{n}=A_{1}^{n} .
\end{gathered}
$$

$A_{n}$ in (13) is the coefficient matrix of $Z_{n}$ which is expressed by $Z_{0}$. If $A_{1}$ is expressed by its characteristic values and characteristic vectors, $A_{1}^{n}$ can be calculated as

$$
\begin{aligned}
& A_{1}=P\left[\begin{array}{cc}
\lambda_{1} & 0 \\
0 & \lambda_{2}
\end{array}\right] P^{-1}, \\
& A_{1}^{n}=P\left[\begin{array}{cc}
\lambda_{1}^{n} & 0 \\
0 & \lambda_{2}^{n}
\end{array}\right] P^{-1} .
\end{aligned}
$$

The characteristic values of $A_{1}$ and its characteristic vectors are

$$
\begin{gathered}
\lambda_{1}=\frac{2 Z_{a}+Z_{b}+\sqrt{Z_{b}^{2}+4 Z_{a} \cdot Z_{b}}}{2}, \\
\lambda_{2}=\frac{2 Z_{a}+Z_{b}-\sqrt{Z_{b}^{2}+4 Z_{a} \cdot Z_{b}}}{2}, \\
P=\left[\begin{array}{cc}
Z_{a} \cdot Z_{b} & \lambda_{2}-Z_{a}-Z_{b} \\
\lambda_{1}-Z_{a} & 1
\end{array}\right], \\
P^{-1}=\left[\begin{array}{cc}
\frac{1}{Z_{b} \cdot\left(\lambda_{1}+Z_{a}\right)} & -\frac{\lambda_{2}-Z_{a}-Z_{b}}{Z_{b} \cdot\left(\lambda_{1}+Z_{a}\right)} \\
-\frac{\lambda_{1}-Z_{a}}{Z_{b} \cdot\left(\lambda_{1}+Z_{a}\right)} & \frac{Z_{a} \cdot Z_{b}}{Z_{b} \cdot\left(\lambda_{1}+Z_{a}\right)}
\end{array}\right] .
\end{gathered}
$$

Combining (16) into (15) results in

$$
\begin{aligned}
A_{1}^{n} & \quad\left[\begin{array}{ll}
\frac{Z_{a} Z_{b} \lambda_{1}^{n}+\lambda_{1} Z_{a} \lambda_{2}^{n}}{Z_{b} \cdot\left(\lambda_{1}+Z_{a}\right)} & \frac{Z_{a} Z_{b}\left(Z_{a}+Z_{b}-\lambda_{2}\right)\left(\lambda_{1}^{n}-\lambda_{2}^{n}\right)}{Z_{b} \cdot\left(\lambda_{1}+Z_{a}\right)} \\
\frac{\left(\lambda_{1}-Z_{a}\right)\left(\lambda_{1}^{n}-\lambda_{2}^{n}\right)}{Z_{b} \cdot\left(\lambda_{1}+Z_{a}\right)} & \frac{\lambda_{1} Z_{b} \lambda_{1}^{n}+Z_{a} Z_{b} \lambda_{2}^{n}}{Z_{b} \cdot\left(\lambda_{1}+Z_{a}\right)}
\end{array}\right], \\
Z_{n}= & \left(\left(Z_{a} Z_{b} \lambda_{1}^{n}+\lambda_{1} Z_{a} \lambda_{2}^{n}\right) \cdot Z_{0}\right. \\
& \left.+Z_{a} Z_{b}\left(Z_{a}+Z_{b}-\lambda_{2}\right)\left(\lambda_{1}^{n}-\lambda_{2}^{n}\right)\right) \\
& \times\left(\left(\lambda_{1}-Z_{a}\right)\left(\lambda_{1}^{n}-\lambda_{2}^{n}\right) Z_{0}+\left(\lambda_{1} Z_{b} \lambda_{1}^{n}+Z_{a} Z_{b} \lambda_{2}^{n}\right)\right)^{-1}
\end{aligned}
$$

where

$$
\begin{gathered}
\lambda_{1}+\lambda_{2}=2 Z_{a}+Z_{b}, \\
\lambda_{1} \cdot \lambda_{2}=Z_{a}^{2} .
\end{gathered}
$$

If $Z_{0}=Z_{a}$, (18) can be simplified using (19) and as

$$
Z_{n}=\frac{\left(Z_{a}-\lambda_{2}\right) \cdot \lambda_{1}^{n+1}-\left(Z_{a}-\lambda_{1}\right) \cdot \lambda_{2}^{n+1}}{\lambda_{1}^{n+1}-\lambda_{2}^{n+1}} .
$$

It is assumed that

$$
\frac{\lambda_{2}}{\lambda_{1}}=\frac{2 Z_{a}+Z_{b}-\sqrt{Z_{b}^{2}+4 Z_{a} \cdot Z_{b}}}{2 Z_{a}+Z_{b}+\sqrt{Z_{b}^{2}+4 Z_{a} \cdot Z_{b}}}=\left|\frac{\lambda_{2}}{\lambda_{1}}\right| e^{j \theta} .
$$

Therefore,

$$
Z_{n}=\frac{\left(Z_{a}-\lambda_{2}\right)-\left(Z_{a}-\lambda_{1}\right) \cdot\left|\lambda_{2} / \lambda_{1}\right|^{n+1} e^{j(n+1) \theta}}{1-\left|\lambda_{2} / \lambda_{1}\right|^{n+1} e^{j(n+1) \theta}} .
$$

If $\left|\lambda_{2} / \lambda_{1}\right|>1$, then the $\lim _{n \rightarrow \infty}\left|\lambda_{2} / \lambda_{1}\right|^{n+1} \rightarrow \infty$,

$$
\lim _{n \rightarrow \infty} Z_{n}=Z_{a}-\lambda_{1}=\frac{-Z_{b}-\sqrt{Z_{b}^{2}+4 Z_{a} \cdot Z_{b}}}{2} .
$$




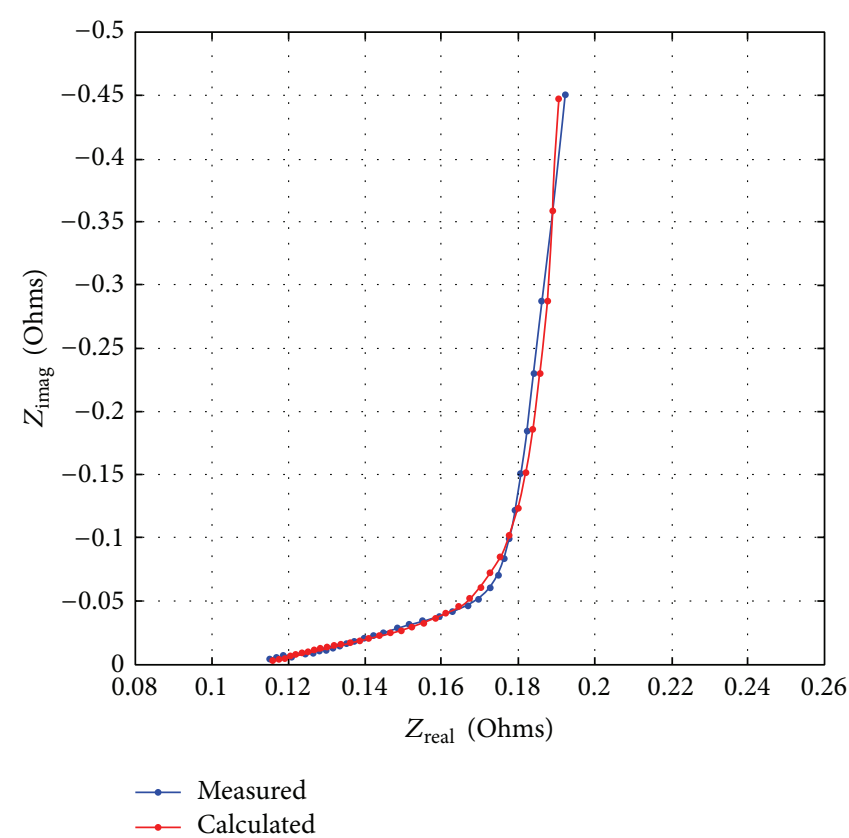

(a) Nyquist plot of a $3.3 \mathrm{~F}$ ultracapacitor (values for the model calculation: $R_{1}=0.1151 \mathrm{Ohm}, C_{1}=3.634 \mathrm{~F}, R_{2}=0.08732 \mathrm{Ohm}, R=0.004589 \mathrm{Ohm}$, and $C=0.03791 \mathrm{~F}$ )

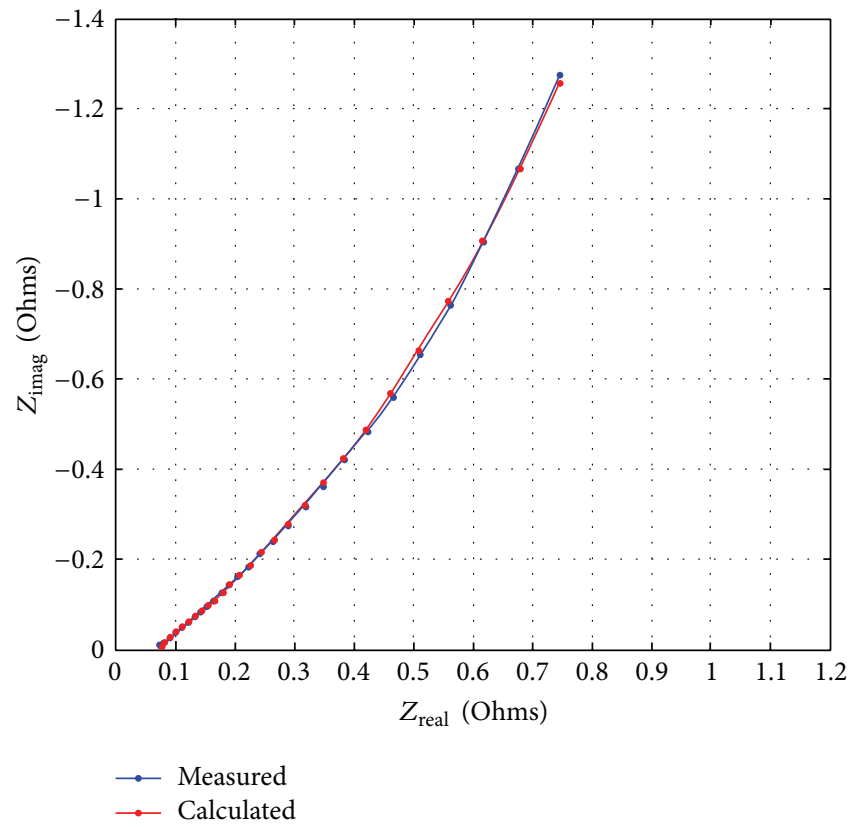

(b) Nyquist plot of a $2 \mathrm{~F}$ ultracapacitor (values for the model calculation: $R_{1}=$ $0.07529 \mathrm{Ohm}, C_{1}=2.063 \mathrm{~F}, R_{2}=3.564 \mathrm{Ohm}, R=0.02241 \mathrm{Ohm}$, and $\mathrm{C}=$ $0.03445 \mathrm{~F})$

FIGURE 14: The Comparison between measured numbers and calculated numbers in Nyquist plot.

Or else, if $\left|\lambda_{2} / \lambda_{1}\right|<1$, then the $\lim _{n \rightarrow \infty}\left|\lambda_{2} / \lambda_{1}\right|^{n+1} \rightarrow 0$,

$$
\lim _{n \rightarrow \infty} Z_{n}=Z_{a}-\lambda_{2}=\frac{-Z_{b}+\sqrt{Z_{b}^{2}+4 Z_{a} \cdot Z_{b}}}{2} .
$$

For the porous electrode model, $Z_{a}=1 / j \omega C, Z_{b}=R$.

In this case, $\left|\lambda_{2} / \lambda_{1}\right|<1$, and (24) applies. Therefore, the formula for the electrode impedance $Z_{a_{0} b_{0}}$ in Figure 10 is

$$
\lim _{n \rightarrow \infty} Z_{a_{0} b_{0}}=-\frac{R}{2}+\sqrt{\frac{R^{2}}{4}+\frac{R}{j \omega C}}
$$

and the input impedance of the ultracapacitor model in Figure 11 is

$$
\begin{aligned}
Z_{a b} & =R_{1}+\frac{1}{j \omega C_{1}}+\frac{1}{1 / R_{2}+1 / Z_{a_{0} b_{0}}} \\
& =R_{1}+\frac{1}{j \omega C_{1}}+\frac{1}{1 / R_{2}+1 /\left(-R / 2+\sqrt{R^{2} / 4+R / j \omega C}\right)} .
\end{aligned}
$$

\section{Validation of Model}

Electrochemical impedance spectroscopy (EIS) is one of the most frequently used analytical tools for the characterization of ultracapacitors. This method was used to determine input resistance and reactance of the ultracapacitor as a function of frequency at a given excitation voltage.
Two ultracapacitors with nominal values of $3.3 \mathrm{~F}$ and $2 \mathrm{~F}$ were measured at room temperature. Potentiostat cycling tests were performed in the capacitor designing lab of St. Jude Medical's Cardiac Rhythm Manufacture, the Liberty, S.C., with a Gamry potentiostat (Reference 3000). The impedance was measured by applying a sinusoidal $5 \mathrm{mV}$ excitation superimposed on a $1.2 \mathrm{~V} \mathrm{DC}$ bias to the ultracapacitor and measuring the magnitude and phase of the current. The frequency range was from $100 \mathrm{mHz}$ to $1 \mathrm{kHz}$.

In this study, a Panasonic 3.3 F/2.3 V (EEC-HW0D335) ultracapacitor which is a new one and a Taiyo Yuden $2 \mathrm{~F} / 2.3 \mathrm{~V}$ (PAS1016LR2R3205) which had been charged and discharged for many times were measured. Figures 14(a) and 14(b) show the magnitude of the input impedance plotted as a function of the real part of the input impedance for the two ultracapacitors. At frequencies above $100 \mathrm{~Hz}$, the electrical behavior of the ultracapacitors is more like a simple resistor than a capacitor. At low frequencies $(f<1 \mathrm{~Hz})$, the imaginary part of the impedance dominates.

The values of $R_{1}, C_{1}, R_{2}, R$, and $C$ for each capacitor model were obtained by curve-fitting the measured data to the model input impedance in (26). It seems that the $R_{2}$ value of old one $(2 \mathrm{~F})$ is larger than the new one $(3.3 \mathrm{~F})$. The experimental data curves of the real part and the imaginary part of $Z_{a b}$ were, respectively, fit using the MATLAB/CF Tool software. Figure 14 also shows the impedance obtained from the model in Figure 11. The plots show excellent agreement between the model and the measured values. Figure 15 shows the measured impedance of a new KAMCAP $400 \mathrm{~F} / 2.7 \mathrm{~V}$ (HP-2R7-J407UY LL) ultracapacitor with a DC bias voltage of $1.2 \mathrm{~V}$ and $10 \mathrm{mV}$ disturbance variable from $100 \mathrm{mHz}$ to $100 \mathrm{~Hz}$ 


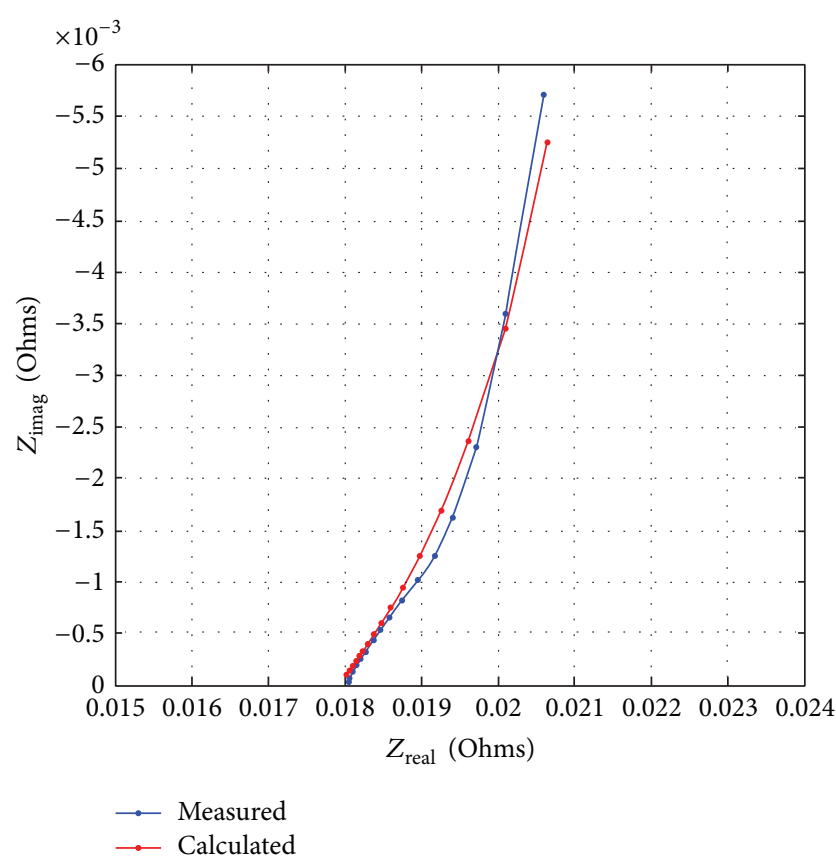

FIgURE 15: Complex-plane representation of a $400 \mathrm{~F}$ ultracapacitor (values for the model calculation: $R_{1}=0.01798 \mathrm{Ohm}, C_{1}=420.5 \mathrm{~F}$, $R_{2}=0.007511 \mathrm{Ohm}, R=0.0001291 \mathrm{Ohm}$, and $\left.C=10 \mathrm{~F}\right)$.

at $+25^{\circ} \mathrm{C}$. This experiment was carried out by Zahner IM6eX. The model data is also shown in Figure 15. In this model $R_{1}=0.01798 \mathrm{Ohm}, C_{1}=420.5 \mathrm{~F}, R_{2}=0.007511 \mathrm{Ohm}$, $R=0.0001291 \mathrm{Ohm}$, and $C=10 \mathrm{~F}$. It also shows excellent agreement between the measured and modeled values.

\section{Conclusion}

A new model for describing the electrical behavior of ultracapacitors is introduced based on an intuitive representation of the electrode pores as a fractal structure. The new model has an infinite number of elements but is fully represented by only five real numbers. A closed-form expression for the input impedance was derived making it relatively easy to fit measured results to the model.

\section{Conflict of Interests}

The authors declare that there is no conflict of interests regarding the publication of this paper.

\section{Acknowledgment}

This work is supported by the Fundamental Research Funds for the Central Universities (no. 2014JBZ017).

\section{References}

[1] P. Barrade, "Series connection of supercapacitors: comparative study of solutions for the active equalization of the voltages," in Proceedings of the 7th International Conference on Modeling and Simulation of Electric Machines, Converters and Systems Electrimacs, Montréal, Canada, August 2002.

[2] J. Van Mierlo, P. Van den Bossche, and G. Maggetto, "Models of energy sources for EV and HEV: Fuel cells, batteries, ultracapacitors, flywheels and engine-generators," Journal of Power Sources, vol. 128, no. 1, pp. 76-89, 2004.

[3] R. Kötz and M. Carlen, "Principles and applications of electrochemical capacitors," Electrochimica Acta, vol. 45, no. 15-16, pp. 2483-2498, 2000.

[4] H. Gualous, D. Bouquain, A. Berthon, and J. M. Kauffmann, "Experimental study of supercapacitor serial resistance and capacitance variations with temperature," Journal of Power Sources, vol. 123, no. 1, pp. 86-93, 2003.

[5] P. L. Taberna, P. Simon, and J. F. Fauvarque, "Electrochemical characteristics and impedance spectroscopy studies of carboncarbon supercapacitors," Journal of the Electrochemical Society, vol. 150, no. 3, pp. A292-A300, 2003.

[6] B. E. Conway, Electrochemical Supercapacitors, Kluwer Academic Publishers/Plenum Press, New York, NY, USA, 1999.

[7] D. Qu and H. Shi, "Studies of activated carbons used in doublelayer capacitors," Journal of Power Sources, vol. 74, no. 1, pp. 99107, 1998.

[8] R. De Levie, in Advancesin Electrochemistry and Electrochemical Engineering, P. Delahay and C. T. Tobias, Eds., vol. 6, pp. 329397, John Wiley \& Sons, New York, NY, USA, 1967.

[9] L. Zubieta and R. Bonert, "Characterization of Double-Layer Capacitors (DLCs) for power electronics applications," in Proceedings of the IEEE Industry Applications Conference, pp. 11491154, October 1998.

[10] F. Rafik, H. Gualous, R. Gallay, A. Crausaz, and A. Berthon, "Frequency, thermal and voltage supercapacitor characterization and modeling," Journal of Power Sources, vol. 165, no. 2, pp. 928-934, 2007.

[11] E. Karden, S. Buller, and R. W. De Doncker, "A frequencydomain approach to dynamical modeling of electrochemical power sources," Electrochimica Acta, vol. 47, no. 13-14, pp. 23472356, 2002.

[12] S. Buller, E. Karden, D. Kok, and R. W. de Doncker, "Modeling the dynamic behavior of supercapacitors using impedance spectroscopy," IEEE Transactions on Industry Applications, vol. 38, no. 6, pp. 1622-1626, 2002.

[13] D. Riu, N. Retière, and D. Linzen, "Half-order modelling of supercapacitors," in Proceedings of the 39th IAS Annual Meeting on Record of the Industry Applications Conference, vol. 4, pp. 2550-2554, IEEE, October 2004.

[14] M. Itagaki, S. Suzuki, I. Shitanda, K. Watanabe, and H. Nakazawa, "Impedance analysis on electric double layer capacitor with transmission line model," Journal of Power Sources, vol. 164, no. 1, pp. 415-424, 2007.

[15] M. Spandana and A. Jagruthi, "Energy Storage Ultracapacitors," Yuva Engineers, 2nd BTech, EEE, 2010.

[16] X. Yuan, "Extraction of the general term formula of fractional recursive sequence of number- $x n=\left(a x_{n-1}+b\right) /\left(c x_{n-1}+d\right)$ by applying matrix method," Journal of China West Normal University (Natural Sciences), vol. 26, no. 1, pp. 102-104, 2005.

[17] Y. Jin and Q. Wang, "The universal solution of ladder network equivalent impedance," Journal of Higher Correspondence Education, vol. 23, no. 4, 2010. 


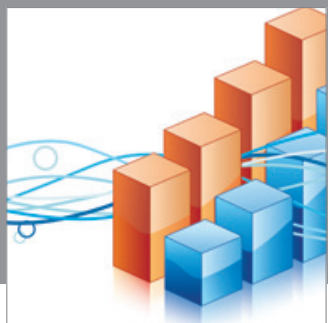

Advances in

Operations Research

mansans

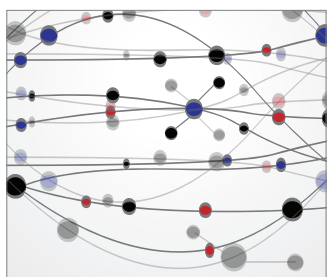

The Scientific World Journal
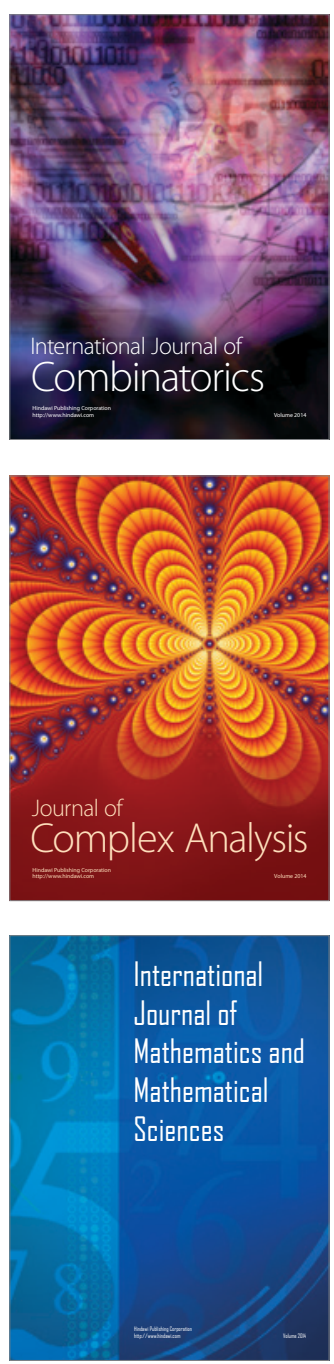
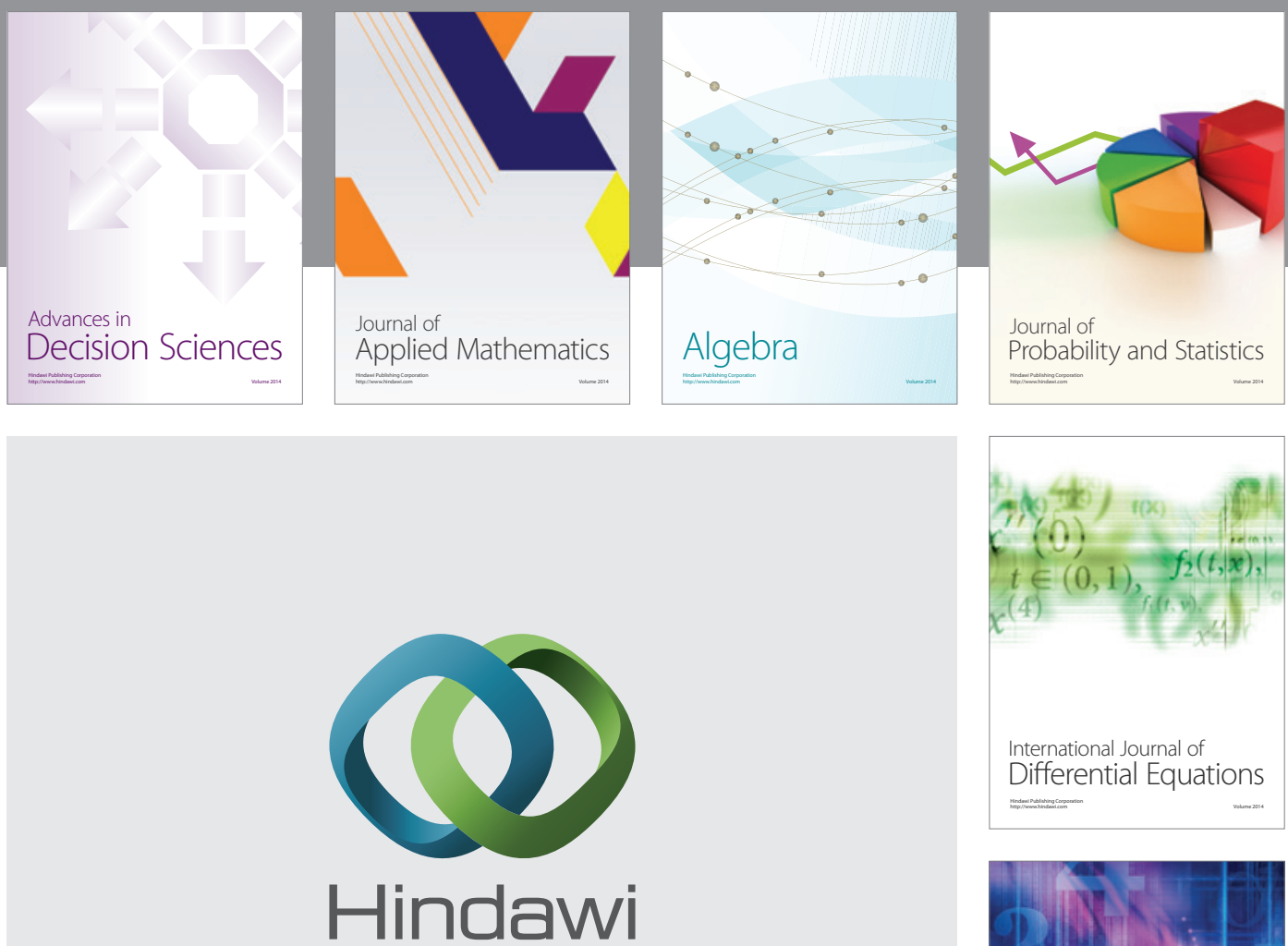

Submit your manuscripts at http://www.hindawi.com
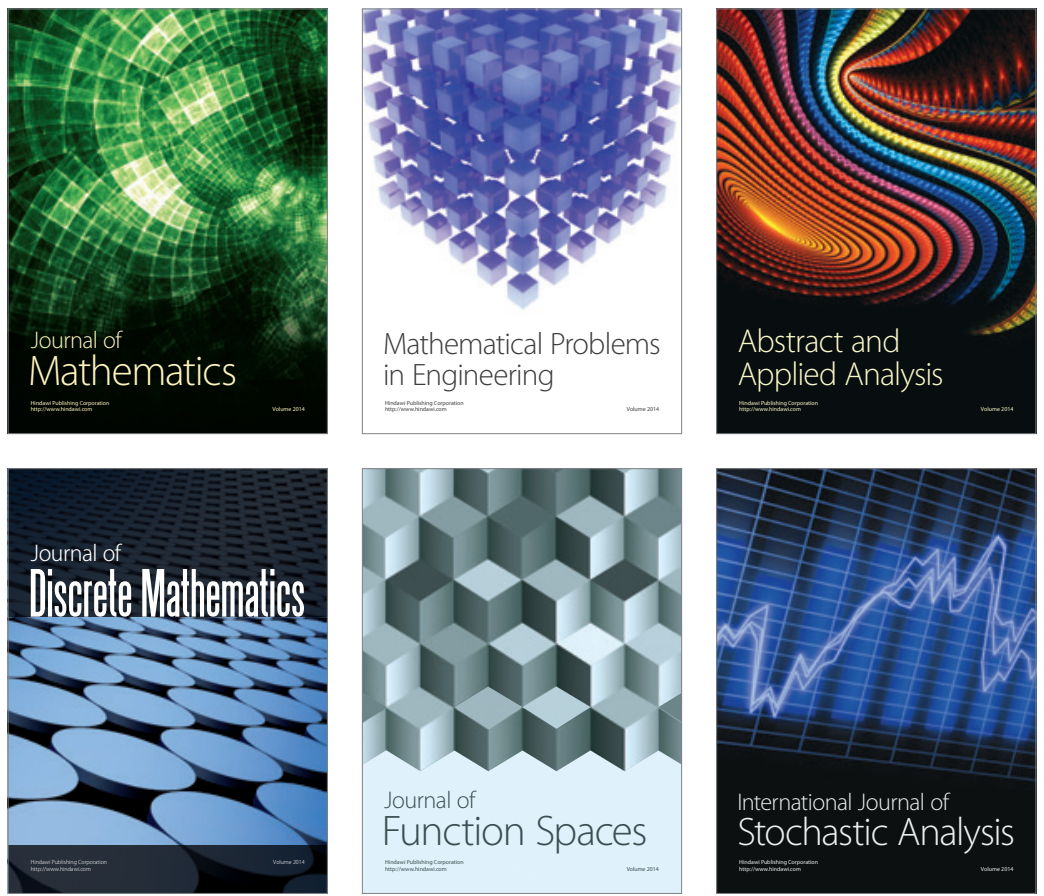

Journal of

Function Spaces

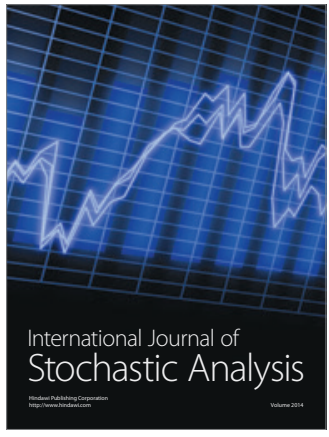

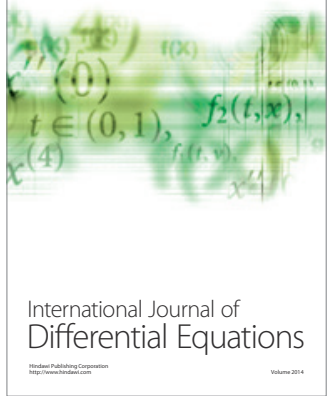
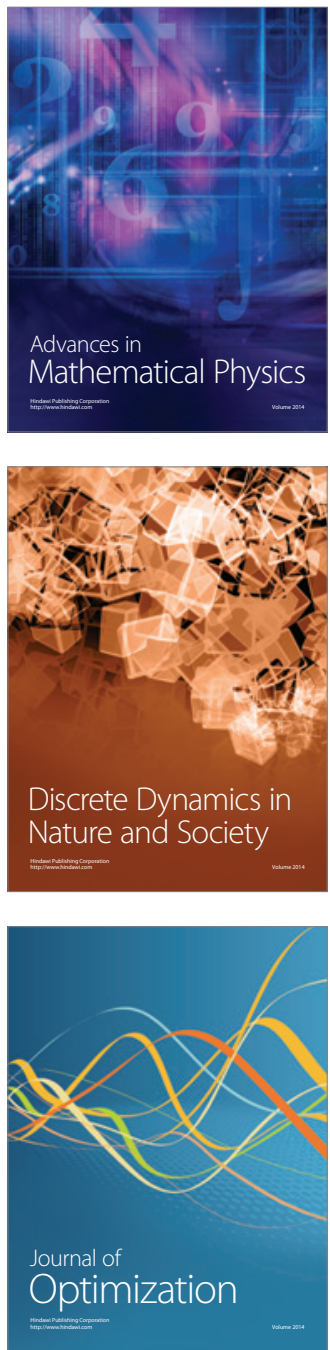